\title{
Stellate Neurons Mediate Functional Hyperemia in the Cerebellar Molecular Layer
}

\author{
Guang Yang, ${ }^{1}$ Josee M. T. Huard, ${ }^{1}$ Alvin J. Beitz, ${ }^{2}$ M. Elizabeth Ross, ${ }^{1}$ and Costantino ladecola ${ }^{1}$ \\ ${ }^{1}$ Center for Clinical and Molecular Neurobiology, Department of Neurology, School of Medicine, and 2Department of \\ Veterinary Pathobiology, School of Veterinary Medicine, University of Minnesota, Minneapolis, Minnesota 55455
}

\begin{abstract}
Mice lacking cyclin D2 have a profound reduction in the number of stellate neurons in the cerebellar molecular layer. We used cyclin D2-null mice to study the contribution of stellate neurons in the increase of cerebellar blood flow (BFcrb) produced by neural activation. Crus II, a region of the cerebellar cortex that receives trigeminal sensory afferents, was activated by stimulation of the upper lip (5-30 V; $10 \mathrm{~Hz})$, and BFcrb was recorded at the activated site by the use of a laser-Doppler flow probe. In wild-type mice, upper lip stimulation increased BFcrb in crus II by $32 \pm 2 \%$. The rise in BFcrb was attenuated by $19 \%$ in heterozygous mice and by $69 \%$ in homozygous mice. In contrast to the cerebellum, the increases in somatosensory cortex blood flow produced by upper lip stimulation was not attenuated in D2-null
\end{abstract}

mice. The field potentials evoked in crus II by upper lip stimulation did not differ between wild-type and D2-null mice. Stellate neurons are a major source of nitric oxide (NO) in the cerebellar molecular layer. The neuronal NO synthase inhibitor 7-nitroindazole attenuated the vascular response to crus II activation in wild-type mice but not in D2-null mice, suggesting that stellate neurons are the major source of NO mediating the vascular response. The data provide evidence that stellate neurons are a critical link between neural activity and blood flow in the activated cerebellum and that NO is the principal effector of their vascular actions.

Key words: cerebral circulation; cerebellum; laser-Doppler flowmetry; vasodilation; glutamate; nitric oxide
Neural activity is a major factor controlling cerebral blood flow (CBF) (for review, see Edvinsson et al., 1993). Although in the resting brain regional $\mathrm{CBF}$ varies in a manner proportional to local levels of neural activity (Reivich, 1974; Lou et al., 1987), during brain activation CBF rises in proportion to the intensity of the stimulation (Fox and Raichle, 1984; Sadato et al., 1997; for review, see Raichle, 1987). The increases in CBF are time-locked to the period of activation and are restricted to the activated sites (Greenberg et al., 1979; Cox et al., 1993). The remarkable spatial and temporal correspondence between brain activity and CBF has provided the opportunity to use activity-induced cerebrovascular changes to localize brain function in humans (Lassen et al., 1978; Raichle, 1998).

However, fundamental issues concerning the relationship between neural activity and CBF still remain to be addressed. For example, it has not been established whether all activated neurons in a given brain region contribute equally to the increase in flow or whether there is a subgroup of cells that is dedicated to neurovascular coupling (Reis, 1984; Lou et al., 1987). Furthermore, the mediators responsible for the vasodilation remain to be identified (Iadecola, 1993; Woolsey et al., 1996). Nitric oxide (NO), a potent vasodilator released during synaptic activity, has emerged recently as an important factor in the mechanisms of functional hyperemia (Gally et al., 1990; Iadecola, 1993). The evidence of the involvement of NO is particularly compelling in cerebellar cortex (Akgören et al., 1994; Yang et al., 1999), wherein NO is thought to play a role in synaptic signaling (Shibuki and Okada, 1991; LevRam et al., 1995; for review, see Daniel et al., 1998). Stimulation of the upper lip in rodents activates somatosensory inputs terminating in a region of the posterior lobe of the cerebellum termed crus II (Welker, 1987; Voogd and Glickstein, 1998). Activation of crus II

Received May 30, 2000; revised June 22, 2000; accepted June 26, 2000.

This work was supported by National Institutes of Health Grants NS 31318 and NS 38252. C.I. is the recipient of a Javits Award from the National Institutes of Health, National Institute of Neurological Diseases and Stroke. The editorial assistance of Tim Murphy is gratefully acknowledged.

Correspondence should be addressed to Dr. C. Iadecola, Department of Neurology, University of Minnesota, 420 Delaware Street Southeast, Minneapolis, MN 55455. E-mail: iadec001@tc.umn.edu. Reprint requests should be addressed to Drs. C. Iadecola or M. E. Ross (E-mail: rossx001@tc.umn.edu) at the above address. Copyright (C) 2000 Society for Neuroscience 0270-6474/00/206968-06\$15.00/0 by perioral stimulation produces increases in cerebellar blood flow (BFcrb) that are restricted to the activated area and are associated with local increases in synaptic activity and glucose use (Yang et al., 1999). The vascular response is substantially reduced by inhibition of neuronal NO synthase (nNOS) (Yang et al., 1999). Therefore, NO is a critical factor in coupling synaptic activity to BFcrb during cerebellar activation. However, despite the importance of $\mathrm{NO}$ in the vascular response to cerebellar activation, its cellular sources have not been defined.

Mice lacking cyclin D2 have a reduced number of stellate neurons in the cerebellar molecular layer (Huard et al., 1999). Therefore, cyclin D2-null mice provide a unique opportunity to study the contribution of stellate neurons to functional hyperemia. Furthermore, considering that stellate neurons are richly endowed with nNOS (Bredt et al., 1990; Vincent and Kimura, 1992), cyclin D2-null mice may provide insight into the sources of NO mediating the vascular response. In this study, therefore, we used cyclin D2-null mice to investigate the role of stellate neurons in the mechanisms of functional hyperemia in the cerebellum.

\section{MATERIALS AND METHODS}

\section{General surgical procedures}

Studies were performed in 2- to 3-month-old cyclin D2-null mice that were obtained from an in-house colony (Huard et al., 1999). All mice were genotyped as described previously (Huard et al., 1999). Homozygous $(-/-)$, heterozygous $(+/-)$, and wild-type $(+/+)$ littermates were studied. Mice were anesthetized with 5\% halothane in 100\% oxygen. After induction of anesthesia, the concentration of halothane was reduced to $1-2 \%$. Catheters were inserted in the femoral artery [polyethylene tubing $10(\mathrm{PE} 10)]$ and in the trachea (PE90; length, $6 \mathrm{~mm}$ ). Animals were then placed in a stereotaxic frame (Kopf Instruments, Tujunga, CA) mounted on a vibration-free table (Technical Manufacturing Corporation, Peabody, MA). Mice were artificially ventilated with an oxygen-nitrogen mixture by a mechanical ventilator (SAR-830; CWE, Inc., Ardmore, PA). The oxygen concentration in the mixture was adjusted to maintain an arterial $\mathrm{pO}_{2}$ $\left(\mathrm{paO}_{2}\right)$ between 120 and $150 \mathrm{mmHg}$ (Table 1). End-tidal $\mathrm{CO}_{2}$ was continuously monitored by the use of a $\mathrm{CO}_{2}$ analyzer (Capstar-100; CWE, Inc.) (Yang et al., 1998; Niwa et al., 2000). Body temperature was maintained at $37 \pm 0.5^{\circ} \mathrm{C}$ by the use of a heating lamp thermostatically controlled by a rectal probe (model 73A-TA; Yellow Springs Instrument Co., Yellow Springs, $\mathrm{OH}$ ). The arterial catheter was used for continuous recording of arterial pressure and heart rate on a chart recorder (model 716P; Grass, Quincy, MA) and for blood sampling. At the end of the surgical procedures, the halothane concentration was reduced to $1 \%$. Because mice were 
Table 1. Arterial pressure and arterial blood gases in mice subjected to perioral stimulation

\begin{tabular}{|c|c|c|c|c|c|}
\hline & $\begin{array}{l}\text { MAP } \\
(\mathrm{mmHg})\end{array}$ & $\begin{array}{l}\mathrm{paCO}_{2} \\
(\mathrm{mmHg})\end{array}$ & $\begin{array}{l}\mathrm{paO}_{2} \\
(\mathrm{mmHg})\end{array}$ & $\mathrm{pH}$ & $n$ \\
\hline \multicolumn{6}{|l|}{ Crus II } \\
\hline \multicolumn{6}{|c|}{ Stimulation parameters } \\
\hline $\mathrm{D} 2+/+$ & $69 \pm 3$ & $33.8 \pm 0.7$ & $137 \pm 18$ & $7.38 \pm 0.04$ & 6 \\
\hline $\mathrm{D} 2+/-$ & $69 \pm 3$ & $34.1 \pm 0.6$ & $143 \pm 9$ & $7.41 \pm 0.04$ & 6 \\
\hline $\mathrm{D} 2-1-$ & $73 \pm 7$ & $35.0 \pm 1.1$ & $136 \pm 12$ & $7.42 \pm 0.04$ & 6 \\
\hline \multicolumn{6}{|c|}{ Adenosine normocapnia } \\
\hline $\mathrm{D} 2+/+$ & $80 \pm 4$ & $34.7 \pm 0.4$ & $148 \pm 12$ & $7.48 \pm 0.04$ & 5 \\
\hline $\mathrm{D} 2+/-$ & $78 \pm 2$ & $34.8 \pm 0.8$ & $147 \pm 9$ & $7.48 \pm 0.03$ & 5 \\
\hline $\mathrm{D} 2-/-$ & $76 \pm 2$ & $35.0 \pm 1.0$ & $145 \pm 8$ & $7.43 \pm 0.04$ & 5 \\
\hline \multicolumn{6}{|c|}{ Hypercapnia (low) } \\
\hline $\mathrm{D} 2+/+$ & $79 \pm 2$ & $44.5 \pm 1.6^{*}$ & $150 \pm 8$ & $7.20 \pm 0.03^{*}$ & \\
\hline $\mathrm{D} 2+/-$ & $80 \pm 4$ & $46.8 \pm 1.2^{*}$ & $144 \pm 6$ & $7.18 \pm 0.02 *$ & \\
\hline D2-/- & $72 \pm 2$ & $45.3 \pm 1.5^{*}$ & $139 \pm 7$ & $7.17 \pm 0.02^{*}$ & \\
\hline \multicolumn{6}{|c|}{ Hypercapnia (high) } \\
\hline $\mathrm{D} 2+/+$ & $67 \pm 5$ & $57.9 \pm 0.9^{*}$ & $141 \pm 11$ & $7.19 \pm 0.01^{*}$ & \\
\hline $\mathrm{D} 2+/-$ & $71 \pm 3$ & $53.7 \pm 1.8^{*}$ & $147 \pm 4$ & $7.20 \pm 0.01^{*}$ & \\
\hline D2-/- & $71 \pm 6$ & $57.1 \pm 1.9^{*}$ & $148 \pm 15$ & $7.19 \pm 0.04 *$ & \\
\hline \multicolumn{6}{|c|}{ 7-NI D2+/+ } \\
\hline Before & $77 \pm 5$ & $35.2 \pm 0.9$ & $142 \pm 10$ & $7.43 \pm 0.04$ & \\
\hline After & $76 \pm 6$ & $35.9 \pm 1.1$ & $136 \pm 8$ & $7.39 \pm 0.04$ & \\
\hline \multicolumn{6}{|c|}{$7-\mathrm{NI} D 2-/-$} \\
\hline Before & $72 \pm 3$ & $35.2 \pm 1.1$ & $128 \pm 10$ & $7.38 \pm 0.05$ & \\
\hline After & $74 \pm 2$ & $35.4 \pm 0.9$ & $143 \pm 10$ & $7.40 \pm 0.04$ & \\
\hline \multicolumn{6}{|l|}{ Neocortex } \\
\hline \multicolumn{6}{|c|}{ Stimulation parameters } \\
\hline $\mathrm{D} 2+/+$ & $79 \pm 2$ & $34.1 \pm 1.1$ & $150 \pm 7$ & $7.45 \pm 0.03$ & 5 \\
\hline $\mathrm{D} 2-/-$ & $75 \pm 2$ & $34.8 \pm 0.8$ & $156 \pm 5$ & $7.46 \pm 0.04$ & 5 \\
\hline
\end{tabular}

Mean \pm SE; * $p<0.05$ from normocapnia (ANOVA and Tukey's test).

\# Data collected in the same mice in which adenosine was tested.

\& Data collected in the same mice in which stimulation parameters were tested.

MAP, Mean arterial pressure; 7-NI, 7-nitroindazole.

not paralyzed, the adequacy of the level of anesthesia was assessed by testing corneal reflexes and motor responses to tail pinch. Throughout the experiment, two to three samples $(50 \mu \mathrm{l})$ of arterial blood were taken for blood gas analysis. Such blood removal did not affect arterial pressure.

\section{Monitoring of blood flow in the cerebellar cortex}

Techniques used for monitoring BFcrb in anesthetized mice have been described previously (Yang et al., 1998; Niwa et al., 2000). A small hole $(3 \times 3 \mathrm{~mm})$ was drilled in the occipital region to expose crus II, and the dura was carefully removed. The cranial window was continuously superfused with Ringer's solution, $\mathrm{pH} 7.3-7.4\left(37 \mathrm{C}^{\circ}\right)$, at a rate of $0.33 \mathrm{ml} / \mathrm{min}$ (Iadecola et al., 1996a). BFcrb was monitored with a Vasamedic laserDoppler flowmeter (model BPM 403A; Saint Paul, MN). The flow probe (tip diameter, $0.8 \mathrm{~mm}$ ) was mounted on a micromanipulator (Kopf Instruments) and positioned $0.5 \mathrm{~mm}$ above the pial surface. The analog output of the flowmeter was amplified (DC amplifier; model 7P1; Grass) and displayed on the polygraph. Changes in BFcrb were calculated as a percentage of baseline flow. The value for zero flow was determined at the end of the experiment after the heart was stopped with an overdose of halothane.

\section{Cerebellar activation and monitoring of field potentials}

Crus II was activated by electrical stimulation of the upper lip with needle electrodes (distance between electrodes, $0.5 \mathrm{~cm}$ ). Stimuli were negative square waves $(10 \mathrm{~Hz} ; 5-30 \mathrm{~V}$; pulse duration, $0.3 \mathrm{msec})$ delivered from a stimulator (model S88; Grass) through a stimulus isolation unit (model PSIU6; Grass). Field potentials evoked in crus II by upper lip stimulation $(1 / \mathrm{sec} ; 20 \mathrm{~V})$ were recorded by glass micropipettes (tip diameter, $5-10 \mu \mathrm{m})$ filled with $2 \mathrm{M} \mathrm{NaCl}$ (resistance, 2-5 M $\Omega$ ) and inserted at a depth of $300-400 \mu \mathrm{m}$. The signal from the micropipettes was amplified (microelectrode amplifier; model 7P5; Grass), displayed on an oscilloscope, and digitized by the use of a computerized data acquisition system (MacAdios Iijr; GW Instruments, Sommerville, MA). In each trial, 10 traces were acquired, averaged, and stored for off-line analysis (Superscope software; GW Instruments) (Yang et al., 1998). Five trials per mice were performed. Field potentials and BFcrb were recorded in separate groups of mice. In some experiments, the parallel fibers were activated electrically by the use of monopolar tungsten microelectrodes (resistance, $1 \mathrm{M} \Omega$ ) inserted into the molecular layer (Yang et al., 1998). Stimuli were negative square waves (1/sec; $20 \mathrm{~V}$; pulse duration, $0.3 \mathrm{msec}$ ), and a silver wire attached to the occipital muscles served as ground. The field potentials evoked by parallel fiber stimulation were recorded by glass micropipettes inserted into the molecular layer at a depth of $20-30 \mu \mathrm{m}$.

\section{Light and electron microscopy}

Immunohistochemistry for nNOS was performed by the use of reagents and methods identical to those described previously (Iadecola et al., 1993; Huard et al., 1999). Briefly, mice were perfused transcardially with $4 \%$ paraformaldehyde, and brains were removed and embedded in paraffin. Sections $(5 \mu \mathrm{m})$ from the cerebella of D $2+/+$ and $\mathrm{D} 2-/-$ mice were cut sagittally with a microtome, deparaffinized, and processed for immunohistochemistry. Antibodies to nNOS (rabbit polyclonal IgG; Upstate Biotechnology, Lake Placid, NY) were used at a dilution of 1:1000. Some sections were stained with hematoxylin and eosin by the use of conventional methods.

Procedures for electron microscopy were similar to those described previously by our laboratories (Clements et al., 1990; Yang et al., 1998). D2 $+/+$ and D2 $-/-$ mice $(n=3 /$ group $)$ were anesthetized with sodium pentobarbital (105-130 mg/kg, i.p.) and perfused transcardially with PBS followed by $5-10 \mathrm{ml}$ of $2 \%$ paraformaldehyde and $2 \%$ glutaraldehyde in PBS. Cerebella were removed, post-fixed in the same fixative, and cut sagittally (thickness, $50 \mu \mathrm{m}$ ) with a vibratome. Sections were fixed in $2 \%$ osmium tetroxide, dehydrated in graded ethanols, embedded in Polybed resin (Polysciences, Warrington, $\mathrm{PA}$ ), and polymerized between dimethyldichlorosilane-coated slides. After polymerization for $1 \mathrm{~d}$ at $40^{\circ} \mathrm{C}$ and $2 \mathrm{~d}$ at $60^{\circ} \mathrm{C}$, the slides were separated, and tissue sections were examined by light microscopy. Cerebellar folia from crus II of D2 $+/+$ and D2 $-/-$ mice were circumscribed with a diamond scribe and excised. These regions were mounted onto Polybed blocks, trimmed, sectioned (thickness, 90-110 $\mathrm{nm}$ ), stained with uranyl acetate and lead citrate, and examined with a Jeol 1200-EXII transmission electron microscope. Representative sections from D2 +/+ and D2 -/- mice were analyzed. Approximately 25 sections ( 5 from each mouse) and at least 30 fields per section were studied. 


\section{Experimental protocol}

After surgical procedures were completed, the superfusion with Ringer's solution was started, and blood gases were adjusted (Table 1). The electrodes for stimulation of the upper lip were inserted, and the animal was allowed to stabilize for $30 \mathrm{~min}$. Stimulation was started when hemodynamic and respiratory parameters were in a steady state.

Effect of perioral stimulation on BFcrb in crus II of D2-null mice. In these experiments, we investigated the effect of crus II activation by perioral stimulation on local BFcrb. The upper lip was stimulated for 30-40 sec epochs at $10 \mathrm{~Hz}$ and with increasing current intensities $(5-30 \mathrm{~V})$. For each current intensity, the increase in BFcrb was recorded in the ipsilateral crus II. As described in detail elsewhere, crus II activation produces increases in BFcrb that reach a plateau after $\sim 30 \mathrm{sec}$ of stimulation (Yang et al., 1999). The BFcrb increase was measured at the level of the plateau.

Effect of hypercapnia or adenosine on BFcrb in cyclin D2-null mice. In these experiments, the increase in BFcrb produced by systemic hypercapnia and by topical application of the vasodilator adenosine was investigated. Two levels of hypercapnia $\left(\mathrm{pCO}_{2}=40-45\right.$ or $50-60 \mathrm{mmHg}$ ) were produced by introducing $\mathrm{CO}_{2}$ into the circuit of the ventilator. A stable level of hypercapnia was maintained until the BFcrb increase reached a steady state. In experiments in which adenosine was studied, this nucleoside $(100$ and $1000 \mu \mathrm{M})$ was superfused on crus II until the BFcrb increase reached a steady state, which usually took $3-5 \mathrm{~min}$. Then, the superfusing solution was switched back to normal Ringer's solution.

Effect of perioral stimulation on the somatosensory cortex $C B F$ in cyclin D2-null mice. In these experiments we investigated the effect of perioral stimulation on $\mathrm{CBF}$ in the somatosensory cortex. The upper lip was stimulated, and $\mathrm{CBF}$ was recorded in the contralateral somatosensory cortex, as described previously (Yang et al., 1999; Niwa et al., 2000). The stimulation parameters used in these studies $(4-16 \mathrm{~Hz} ; 10-25 \mathrm{~V})$ were found in preliminary investigations to elicit maximal changes in $\mathrm{CBF}$

Effect of 7-nitroindazole on the increases in BFcrb produced by perioral stimulation. To study the role of $\mathrm{NO}$ in the increase of BFcrb produced by perioral stimulation, we used the nNOS inhibitor 7-nitroindazole (7-NI) (Babbedge et al., 1993; Iadecola et al., 1996b). Both D2 +/+ and D2 - /mice were studied. The increase in BFcrb produced by upper lip stimulation $(10-25 \mathrm{~V} ; 10 \mathrm{~Hz})$ was tested before and after administration of 7-NI $(50 \mathrm{mg} / \mathrm{kg}$, i.p.). This concentration of 7-NI inhibits nNOS activity in this preparation by $60 \%$ without affecting endothelial NOS-dependent function (Iadecola et al., 1996b). We have demonstrated previously that this concentration of 7-NI does not influence the field potentials produced by crus II activation (Yang et al., 1999).

\section{Data analysis}

Data in the text, table, and figures are presented as the mean \pm SE. Multiple comparisons were evaluated by the ANOVA and Tukey's test (Systat, Evanston, IL). Two-group comparisons were evaluated by the two-tailed Student's $t$ test. Differences were considered significant for probability values $<0.05$.

\section{RESULTS}

\section{Structure of the cerebellar molecular layer in cyclin D2-null mice}

First, we investigated the morphology of the molecular layer in cyclin D2-null mice by light and electron microscopy (Fig. 1). We used nNOS immunohistochemistry to localize stellate neurons (Bredt et al., 1990; Vincent and Kimura, 1992). In D2 +/+ mice, many nNOS-positive cells with the morphology of stellate neurons were observed in the outer molecular layer (Fig. $1 A, C$ ). In cyclin $\mathrm{D} 2$-null mice, the number of stellate neurons was markedly reduced. The larger nNOS-positive cells located near the Purkinje cell layer have the morphology of basket cells (Fig. 1B,D), which are preserved in cyclin D2-null mice (Huard et al., 1999). At the ultrastructural level, stellate neurons were observed in the superficial regions of the molecular layer in cyclin D $2+/+$ mice and were recognized by their distinctive nuclear morphology, scanty cytoplasm, and paucity of somatic synaptic contacts (Palay and ChanPalay, 1974; Yang et al., 1998). In cyclin D2 -/- mice, stellate neurons were not observed (data not shown). However, the morphology of Purkinje cell dendrites and parallel fiber synapses did not differ between D2 $-/-$ and D2 $+/+$ mice (Fig. $1 E, F$ ). These observations provide further evidence that stellate neurons are depleted in D2 $-/-$ mice, whereas other cells in the molecular layer are not altered.

\section{Effect of perioral stimulation on BFcrb in crus II in D2-null mice}

In $\mathrm{D} 2+/+$ mice, activation of crus II by perioral stimulation increased BFcrb (Fig. 2). The increases in flow were related to the intensity of stimulation and reached a plateau at 25-30 V. In D2 $+/-$ mice, the increases in BFcrb evoked by perioral stimulation in crus II were attenuated, but the reduction did not reach statistical significance $(p>0.05$; ANOVA and Tukey's test). In D2 -/mice, the flow increases were significantly attenuated (Fig. $2 ;-69 \%$ at $30 \mathrm{~V} ; p<0.05)$. In contrast to the hyperemia produced by perioral stimulation, the increases in BFcrb produced by topical superfusion with the vasodilator adenosine $(100$ and $1000 \mu \mathrm{M})$ or by systemic hypercapnia were not affected in D2 $+/+$, D2 +/-, or D2 $-/-$ mice (Fig. 3).

\section{Field potentials in D2-null mice}

To determine whether the attenuation of functional hyperemia in cyclin D2 -/- mice was caused by a reduction in the intensity of neural activation, the field potentials evoked by perioral stimulation were recorded in crus II. Activation of crus II produced the characteristic polyphasic potentials (Chen et al., 1996) (Fig. 4). These potentials were qualitatively similar in D2 +/+, D2 +/-, and D2 $-/-$ mice (Fig. 4, top; $n=3$ /group). In addition, the field potentials produced by local electrical stimulation of the parallel fibers (Eccles et al., 1966; Ito, 1984) did not differ in D2 +/+, D2 $+/-$, and D2 -/- mice (Fig. 4, bottom).

\section{Effect of perioral stimulation on CBF in the somatosensory cortex in D2-null mice}

In these experiments, we investigated the effect of perioral stimulation on $\mathrm{CBF}$ in the somatosensory cortex. In $\mathrm{D} 2+/+$ mice, perioral stimulation increased $\mathrm{CBF}$ in the somatosensory cortex. The magnitude of the increases in $\mathrm{CBF}$ was related to the frequency and intensity of stimulation (Fig. 5). In contrast to crus II, the increases in somatosensory cortex blood flow produced by perioral stimulation did not differ between cyclin D2 $+/+$ and D2 $-/-$ mice (Fig. $5 ; p>0.05$ ).

\section{Effect of 7-NI on the increase in BFcrb produced by perioral stimulation}

As shown above, cyclin D2 -/- mice have a paucity of stellate neurons and exhibit a reduction in the hyperemic response produced by crus II activation. Because stellate neurons are a major source of nNOS in the molecular layer (Bredt et al., 1990; Southam et al., 1992; Vincent and Kimura, 1992), it is likely that the component of the increase in BFcrb that depends on stellate neurons is mediated by NO. To test this hypothesis, we studied the effect of the nNOS inhibitor 7-NI on the increase in BFcrb produced by perioral stimulation. In D $2+/+$ mice $7-\mathrm{NI}$ attenuated the response substantially (Fig. 6). However, in D2 -/- mice 7-NI did not reduce the rise in BFcrb (Fig. 6). Therefore, nNOS inhibition does not attenuate further the BFcrb response in D2 $-/-$ mice.

\section{DISCUSSION}

We used cyclin D2-null mice to gain an insight into the cellular mechanisms responsible for the increase in BFcrb produced by synaptic activity in the cerebellar cortex. Cyclin D2-null mice lack the cell cycle protein cyclin D2 and have a marked reduction in the number of stellate neurons in the cerebellar molecular layer (Huard et al., 1999). The cerebellum of D2-null mice is smaller than that of wild-type littermates, a finding that can be attributed to a reduced number of granule neurons (Huard et al., 1999). However, D2-null mice do not have overt motor alterations suggestive of cerebellar dysf unction (Huard et al., 1999). The molecular basis of the cerebellar alterations observed in D2-null mice is unknown, but these alterations are thought to be related to the role of cell cycle proteins in primary neurogenesis and differentiation (Ross, 1996). In the present study, we first sought to examine in greater detail the morphology of the cerebellar molecular layer in D2-null mice, both at the light and ultrastructural level. We found a reduction in the number of stellate cells but no ultrastructural abnormalities in other cellular elements of the molecular layer. These findings suggest that the paucity of stellate neurons is not associated 

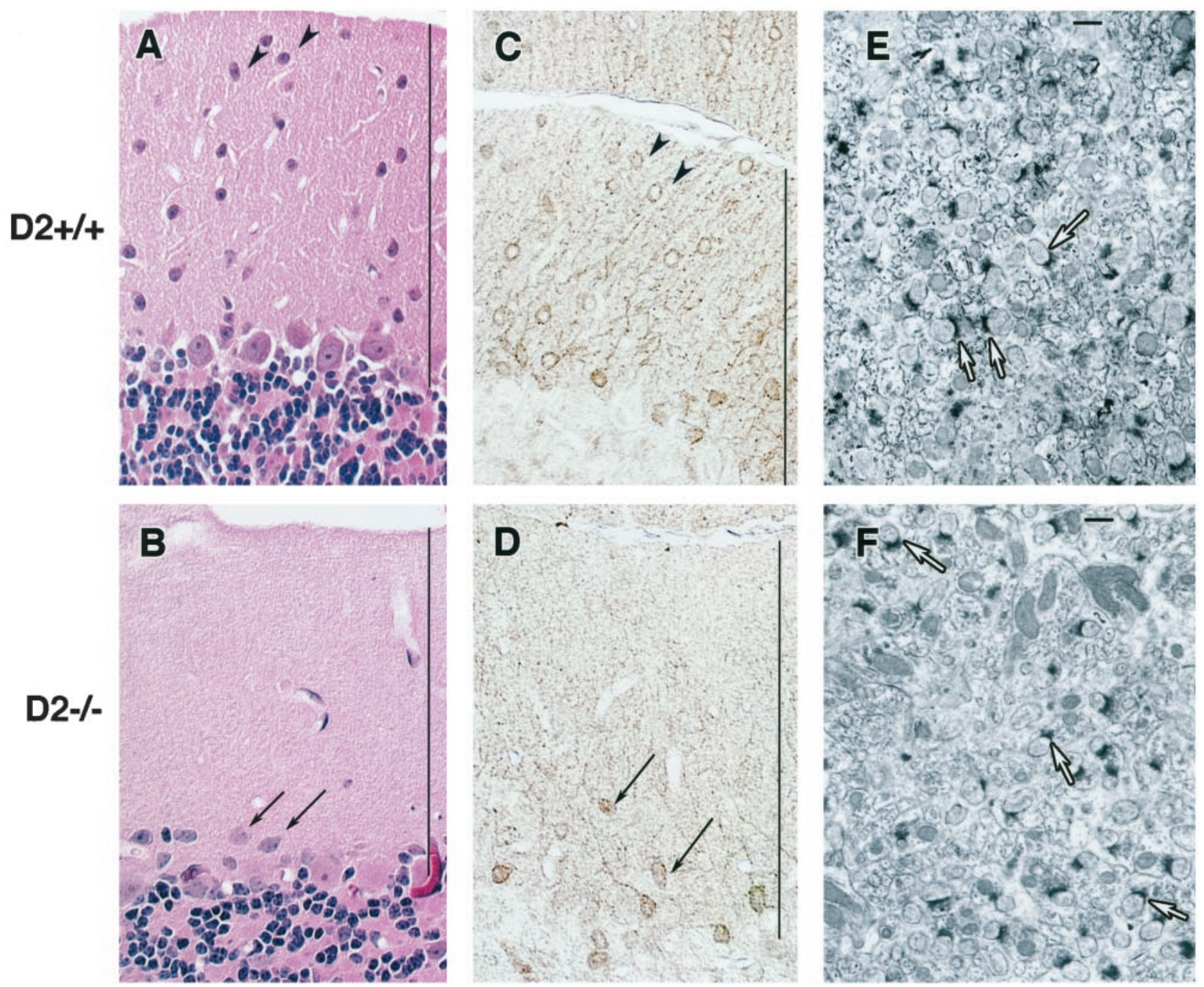

Figure 1. Morphology of the cerebellar molecular layer in cyclin D2-null mice. $A, B$, Hematoxylin and eosin stain of the molecular layer in cyclin D2 $+/+$ $(A)$ and $\mathrm{D} 2-/-(B)$ mice. $C, D$, nNOS immunohistochemistry of the cerebellar molecular layer in $\mathrm{D} 2+/+(C)$ and $\mathrm{D} 2-/-(D)$ mice. Arrowheads in $A$ and $C$ point to nNOS-immunoreactive cells with the morphological characteristics of stellate neurons. Parallel arrows in $B$ and $D$ point to nNOS-immunoreactive cells with the morphological characteristics of basket neurons. $E, F$, Ultrastructure of the cerebellar molecular layer in D2 $+/+(E)$ and D2 $-/-(F)$ mice. Arrows point to asymmetric synapses between parallel fibers and Purkinje cell dendrites. Lines in $A-D$ define the width of the molecular layer. Scale bars (in $E$ and $F$ ): $1 \mu \mathrm{m}$.

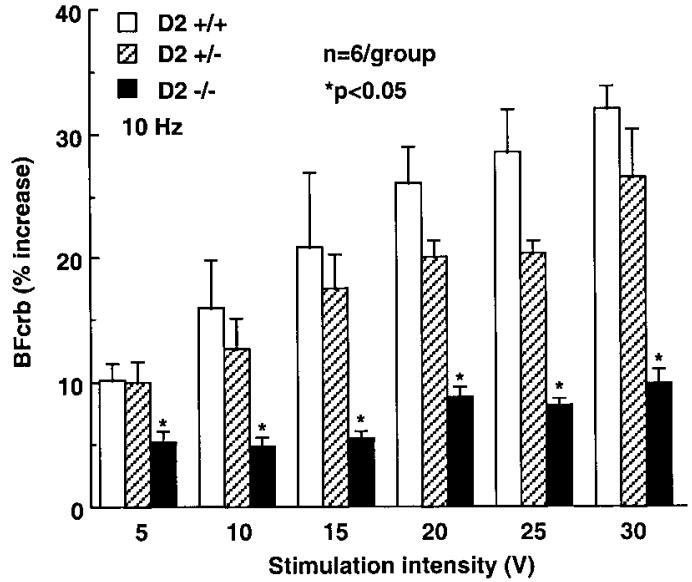

Figure 2. Effect of upper lip stimulation on the evoked elevations in BFcrb in crus II in D2-null mice. The magnitude of the flow increase is attenuated in D2-null mice ( $p<0.05$; ANOVA and Tukey's test).
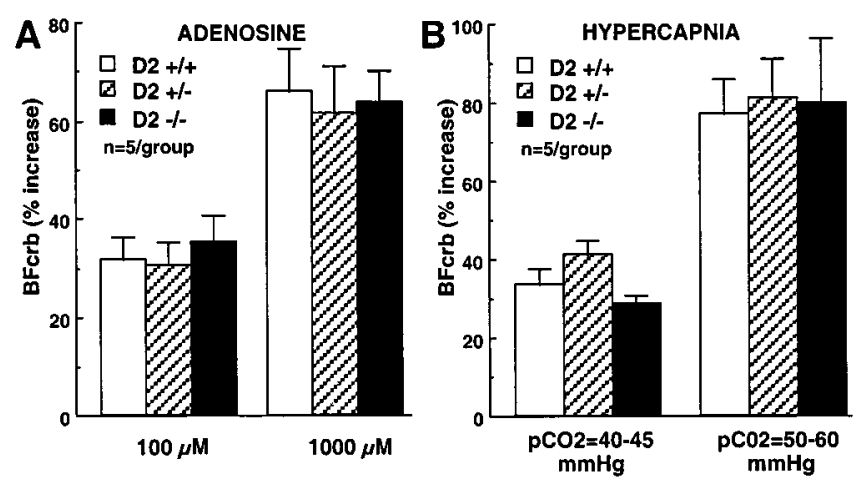

Figure 3. A, Effect of topical application of adenosine on BFcrb in D2-null mice. Adenosine was superfused on crus II. $B$, Effect of systemic hypercapnia on BFcrb in crus II in D2-null mice.

with secondary morphological alterations in the structure of the molecular layer.

We then studied the hemodynamic response evoked by crus II activation in D2-null mice. We found that these mice have a substantial reduction in the BFcrb increase produced by somatosensory activation. In contrast to the cerebellum, the increase in 


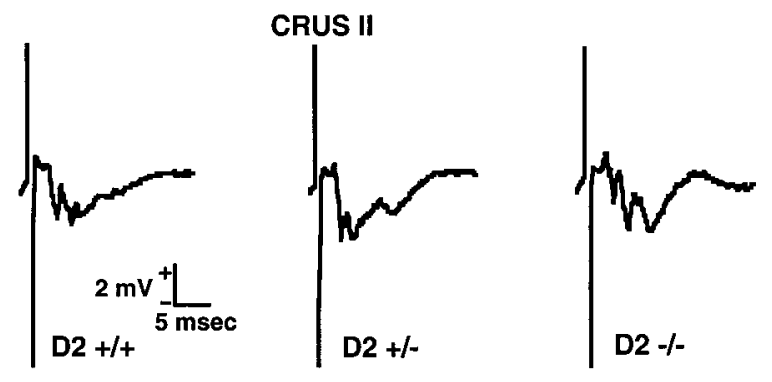

PARALLEL FIBERS
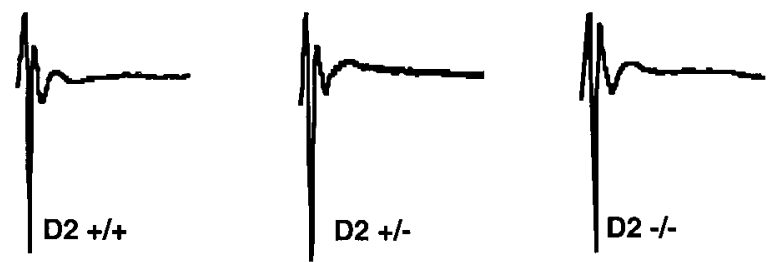

Figure 4. Field potentials evoked in crus II by upper lip stimulation (top) or by direct stimulation of the parallel fibers (bottom).
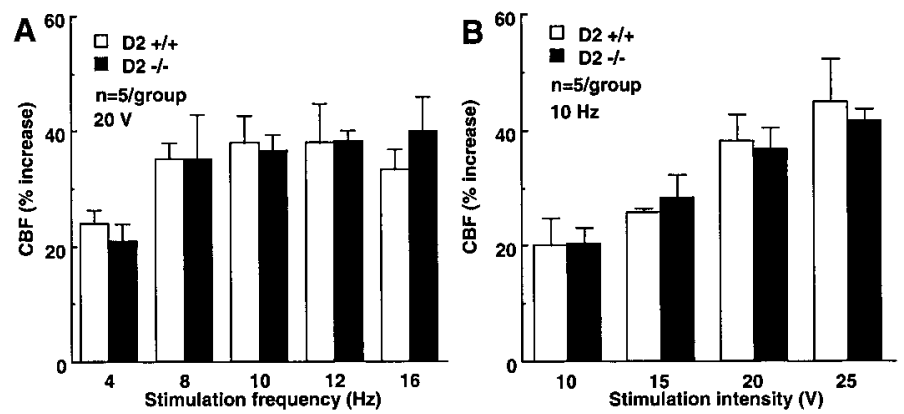

Figure 5. Effect of upper lip stimulation on $\mathrm{CBF}$ in the contralateral somatosensory cortex. $A$, The effect of stimulation frequency. $B$, The effect of stimulation intensity.

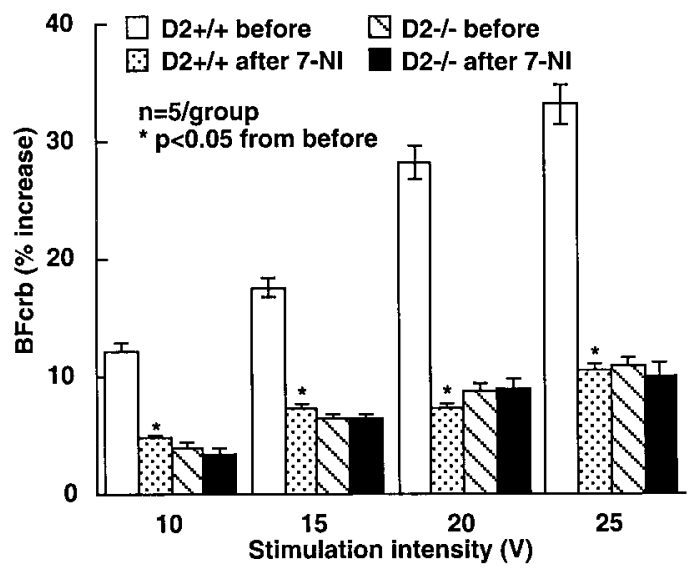

Figure 6. Effect of the nNOS inhibitor 7-NI on the elevations in crus II $\mathrm{BF}$ crb in D2-null mice. 7-NI attenuates the flow increase in D2 $+/+$ but not in $\mathrm{D} 2-/-$ mice.

somatosensory cortex blood flow elicited by the same stimulus was not affected. Therefore, the reduction in functional hyperemia is restricted to the cerebellum and is not observed in other areas receiving the same somatosensory inputs. We also found that the field potentials evoked by crus II activation did not differ between $\mathrm{D} 2+/+$ and D2 $-/-$ mice. This observation suggests that the reduction in the BFcrb response to functional activation is not caused by a reduction in the intensity of the neural activity pro- duced by the stimulation. Furthermore, it indicates that the activity of stellate interneurons does not contribute substantially to the amplitude or shape of the field potentials evoked by activation of crus II. However, a quantitative analysis of the field potentials is needed to establish this point more firmly.

The reduction of the hemodynamic response in $\mathrm{D} 2-/-$ mice cannot result from differences in arterial pressure or blood gases because these variables were caref ully controlled and did not differ among the groups of mice studied. Furthermore, the effect cannot be a consequence of a nonspecific alteration in cerebrovascular reactivity in D2-null mice because the increase in BFcrb produced by other stimuli, such as systemic hypercapnia or topical application of adenosine, was not affected. Therefore, the reduction of the BFcrb response in cyclin D2-null mice cannot be attributed to changes in systemic variables or nonspecific alterations in vascular reactivity.

The vascular response elicited by crus II activation is mediated in large part by NO (Yang et al., 1999). Therefore, in subsequent experiments we investigated the cellular source of NO responsible for the vascular response evoked by crus II activation. In the molecular layer, nNOS is present in parallel fibers, as well as in stellate and basket neurons (Bredt et al., 1990; Southam et al., 1992; Vincent and Kimura, 1992). If stellate neurons are the main source of the NO mediating the vascular response, then inhibition of nNOS should reduce the response in D2 +/+ but not in D2 -/mice. In agreement with this prediction, we found that the nNOS inhibitor 7-NI attenuates the BFcrb response in D2 $+/+$ mice but had no effect in D2 $-/-$ mice.

These observations, collectively, suggest that stellate cells are a critical link between neural activity and blood flow in the cerebellar molecular layer. The most likely scenario is that during upper lip stimulation stellate cells are activated via trigeminal projections to the granule cell-parallel fiber system and to the inferior oliveclimbing fiber system as well (Steindler, 1985; Ikeda and Matsushita, 1992). Glutamate-induced depolarization of stellate neurons increases intracellular calcium, possibly via calcium-permeable AMPA receptors (Liu and Cull-Candy, 2000). Such an increase in intracellular calcium, in turn, activates nNOS, leading to NO production and vasodilation. Therefore, in the cerebellar molecular layer, stellate neurons might be directly involved in coupling synaptic activity to blood flow. However, cyclin D2-null mice also have a reduction in the number of cerebellar granule cells (Huard et al., 1999). Considering that the parallel fibers are axons of granule cells, it is possible that the attenuation in functional hyperemia is caused by a reduction in the number of parallel fibers and their synaptic contacts with Purkinje cell dendrites. However, this is unlikely to be the case. First, we have provided morphological evidence that the synapses between parallel fibers and Purkinje cells are not depleted in the molecular layer of D2-null mice. Second, we have shown that the field potentials evoked by parallel fiber stimulation are not attenuated in D2-null mice. It is, therefore, unlikely that the attenuation of functional hyperemia in D2-null mice is entirely caused by a reduction in the number of parallel fibers. However, a modest contribution from the parallel fibers cannot be excluded.

The increase in CBF produced by hypercapnia was preserved in D2-null mice. Although NO is also involved in the mechanisms of the hypercapnic vasodilation, recent evidence indicates that NO acts as a "permissive" factor facilitating the action of other vasodilators (for review, see Iadecola, 1999). Therefore, one possibility is that in D2-null mice other sources of NO, such as parallel fibers, perivascular nerves, or endothelium, compensate for the lack of NO produced by stellate neurons. Alternatively, factors other than NO could contribute to the hypercapnic vasodilation in cyclin D2-null mice. In contrast to hypercapnia, NO released during synaptic activity is an absolute requirement for the increase in BFcrb produced by cerebellar activation (Yang and Iadecola, 1997; Yang et al., 1999). Therefore, in D2-null mice, other sources of NO cannot restore the increase in BFcrb produced by crus II activation.

The findings of the present study support the hypothesis that there are central neurons dedicated to coupling synaptic activity to 
local blood flow. However, it is unknown whether the cellular mechanisms of functional hyperemia in the cerebellum apply also to other brain regions. In the cerebral cortex, it has been proposed that neurons with preferential association to cerebral blood vessels play a role in flow regulation during neural activity (Iadecola et al., 1993; Vaucher and Hamel, 1995; Krimer et al., 1998). However, direct evidence of a role of these neurons in the regulation of the cerebral circulation in vivo is lacking (Iadecola, 1998).

Another issue concerns the functional consequences of the reduction in functional hyperemia in cyclin D2-null mice. The reduction in the flow response to activation does not interfere with synaptic transmission in the molecular layer, as evidenced by the normal field potentials. Furthermore, the mice do not exhibit evidence of motor abnormalities suggestive of cerebellar dysfunction. However, the long-term consequences of the reduced hyperemic response are not known. It is conceivable that alterations in substrate delivery and waste removal resulting from the reduced flow response may have long-term effects on cerebellar function. These remain to be defined.

In conclusion, we used cyclin D2-null mice to investigate the cellular mechanisms of the in vivo regulation of BFcrb during functional activation of the cerebellar cortex. We found that these mice have a substantial reduction in the number of stellate cells in the cerebellar molecular layer and exhibit a marked attenuation in the increase in crus II blood flow produced by somatosensory stimuli. These vascular effects cannot be attributed to a reduction in the local neural activity evoked by the stimulation or to nonspecific alterations in vascular reactivity in the null mice. We have also provided evidence that the component of the hyperemic response attributable to stellate neurons is mediated by NO. The data suggest a new functional role for stellate neurons and provide an insight into the cellular mechanisms regulating the cerebellar microcirculation during neural activity.

\section{REFERENCES}

Akgören N, Fabricius M, Lauritzen M (1994) Importance of nitric oxide for local increases of blood flow in rat cerebellar cortex during electrical stimulation. Proc Natl Acad Sci USA 91:5903-5907.

Babbedge RC, Bland WP, Hart SL, Moore PK (1993) Inhibition of rat cerebellar nitric oxide synthase by 7-nitroindazole and related substituted indazoles. Br J Pharmacol 110:225-228.

Bredt DS, Hwang PM, Snyder SH (1990) Localization of nitric oxide synthase indicating a neural role for nitric oxide. Nature 347:768-770.

Chen G, Hanson CL, Ebner TJ (1996) Functional parasagittal compartments in the rat cerebellar cortex: an in vivo optical imaging study using neutral red. J Neurophysiol 76:4169-4174.

Clements JR, Magnusson KR, Beitz AJ (1990) Ultrastructural description of glutamate-, aspartate-, taurine-, and glycine-like immunoreactive terminals from five rat brain regions. J Electron Microsc Tech 15:49-66.

Cox SB, Woolsey TA, Rovainen CM (1993) Localized dynamic changes in cortical blood flow with whisker stimulation corresponds to matched vascular and neuronal architecture of rat barrels. J Cereb Blood Flow Metab 13:899-913.

Daniel H, Levenes C, Crepel F (1998) Cellular mechanisms of cerebellar LTD. Trends Neurosci 21:401-407.

Eccles JC, Llinas R, Sasaki K (1966) Parallel fibre stimulation and the responses induced thereby in the Purkinje cells of the cerebellum. Exp Brain Res 1:17-39.

Edvinsson L, MacKenzie ET, McCulloch J (1993) Cerebral blood flow and metabolism, $683 \mathrm{pp}$. New York: Raven.

Fox PT, Raichle ME (1984) Stimulus rate dependence of regional cerebral blood flow in human striate cortex, demonstrated by positron emission tomography. J Neurophysiol 51:1109-1120.

Gally JA, Montague PR, Reeke GNJ, Edelman GM (1990) The NO hypothesis: possible effects of a short-lived, rapidly diffusible signal in the development and function of the nervous system. Proc Natl Acad Sci USA 87:3547-3551.

Greenberg J, Hand P, Sylvestro A, Reivich M (1979) Localized metabolicflow couple during functional activity. Acta Neurol Scand 72:12-13.

Huard JM, Forster CC, Carter ML, Sicinski P, Ross ME (1999) Cerebellar histogenesis is disturbed in mice lacking cyclin D2. Development 126:1927-1935.

Iadecola C (1993) Regulation of the cerebral microcirculation during neural activity: is nitric oxide the missing link? Trends Neurosci 16:206-214.

Iadecola C (1998) Neurogenic control of the cerebral microcirculation: is dopamine minding the store? Nat Neurosci 1:263-265.

Iadecola C (1999) The role of NO in cerebrovascular regulation and stroke. In: (Mathie RT, Griffith TM, eds), pp 202-225. London: Imperial College.

Iadecola C, Beitz AJ, Renno W, Xu X, Mayer B, Zhang F (1993) Nitric oxide synthase-containing neural processes on large cerebral arteries and cerebral microvessels. Brain Res 606:148-155.

Iadecola C, Li J, Yang G, Xu S (1996a) Neural mechanisms of blood flow regulation during synaptic activity in cerebellar cortex. J Neurophysiol 75:940-950.

Iadecola C, Xu S, Yang G (1996b) 7-Nitroindazole attenuates vasodilation from cerebellar parallel fiber stimulation but not acetylcholine. Am J Physiol 270:R914-R919.

Ikeda M, Matsushita M (1992) Trigeminocerebellar projections to the posterior lobe in the cat, as studied by anterograde transport of wheat germ agglutinin-horseradish peroxidase. J Comp Neurol 316:221-237.

Ito $\mathrm{M}$ (1984) The cerebellum and neural control, $580 \mathrm{pp}$. New York: Raven.

Krimer LS, Muly III EC, Williams GV, Goldman-Rakic PS (1998) Dopaminergic regulation of cerebral cortical microcirculation. Nat Neurosci $1: 286-289$.

Lassen NA, Ingvar DH, Skinhoj E (1978) Brain function and blood flow. Sci Am 239:62-71.

Lev-Ram V, Makings LR, Keitz PF, Kao JP, Tsien RY (1995) Long-term depression in cerebellar Purkinje neurons results from coincidence of nitric oxide and depolarization-induced $\mathrm{Ca}^{2+}$ transients. Neuron 15:407-415.

Liu SQ, Cull-Candy SG (2000) Synaptic activity at calcium-permeable AMPA receptors induces a switch in receptor subtype. Nature 405:454-458.

Lou HC, Edvinsson L, MacKenzie ET (1987) The concept of coupling blood flow to brain function: revision required? Ann Neurol 22:289-297.

Niwa K, Araki E, Morham SG, Ross ME, Iadecola C (2000) Cyclooxygenase-2 contributes to functional hyperemia in whisker-barrel cortex. J Neurosci 20:763-770.

Palay SL, Chan-Palay V (1974) Cerebellar cortex, 348 pp. New York: Springer.

Raichle ME (1987) Circulatory and metabolic correlates of brain function in normal humans. In: The nervous system, Vol 5, Higher functions of the brain, Pt 2 (Plum F, ed), pp 643-674. Bethesda, MD: American Physiological Society.

Raichle ME (1998) Behind the scenes of functional brain imaging: a historical and physiological perspective. Proc Natl Acad Sci USA 95:765-772.

Reis D (1984) Central neural control of cerebral circulation and metabolism. In: Neurotransmitters and the cerebral circulation (MacKenzie E, Seylaz J, Bes A, eds), pp 91-119. New York: Raven.

Reivich M (1974) Blood flow metabolism couple in brain. Res Publ Assoc Res Nerv Ment Dis 53:125-140.

Ross ME (1996) Cell division and the nervous system: regulating the cycle from neural differentiation to death. Trends Neurosci 19:62-68.

Sadato N, Ibanez V, Campbell G, Deiber MP, Le Bihan D, Hallett M (1997) Frequency-dependent changes of regional cerebral blood flow during finger movements: functional MRI compared to PET. J Cereb Blood Flow Metab 17:670-679.

Shibuki D, Okada D (1991) Endogenous nitric oxide release required for long-term synaptic depression in the cerebellum. Nature 349:326-328.

Southam E, Morris R, Garthwaite J (1992) Sources and targets of nitric oxide in rat cerebellum. Neurosci Lett 137:241-244.

Steindler DA (1985) Trigeminocerebellar, trigeminotectal, and trigeminothalamic projections: a double retrograde axonal tracing study in the mouse. J Comp Neurol 237:155-175.

Vaucher E, Hamel E (1995) Cholinergic basal forebrain neurons project to cortical microvessels in the rat: electron microscopic study with anterogradely transported Phaseolus vulgaris leucoagglutinin and choline acetyltransferase immunocytochemistry. J Neurosci 15:7427-7441.

Vincent SR, Kimura H (1992) Histochemical mapping of nitric oxide synthase in the rat brain. Neuroscience 46:755-784.

Voogd J, Glickstein M (1998) The anatomy of the cerebellum. Trends Neurosci 21:370-375.

Welker W (1987) Spatial organization of somatosensory projections to granule cells cerebellar cortex: functional and connectional implications of fractured somatotopy (summary of Wisconsin studies). In: New concepts in cerebellar neurobiology (King JS, ed), pp 239-280. New York: Liss.

Woolsey TA, Rovainen CM, Cox SB, Henegar MH, Liang GE, Liu D, Moskalenko YE, Sui J, Wei L (1996) Neuronal units linked to microvascular modules in cerebral cortex: response elements for imaging the brain. Cereb Cortex 6:647-660.

Yang G, Iadecola C (1997) Obligatory role of NO in glutamate-dependent hyperemia evoked from cerebellar parallel fibers. Am J Physiol 272:R1155-R1161.

Yang G, Feddersen RM, Zhang F, Clark HB, Beitz AJ, Iadecola C (1998) Cerebellar vascular and synaptic responses in transgenic mice with Purkinje cell dysfunction. Am J Physiol 274:R529-R540.

Yang G, Chen G, Ebner TJ, Iadecola C (1999) Nitric oxide is the predominant mediator of cerebellar hyperemia during somatosensory activation in rat. Am J Physiol 277:R1760-R1770. 